\title{
Toward an AdS/cold atoms correspondence: A geometric realization of the Schrödinger symmetry
}

\author{
D. T. Son \\ Institute for Nuclear Theory, University of Washington, Seattle, Washington 98195-1550, USA \\ (Received 28 April 2008; published 4 August 2008)
}

\begin{abstract}
We discuss a realization of the nonrelativistic conformal group (the Schrödinger group) as the symmetry of a spacetime. We write down a toy model in which this geometry is a solution to field equations. We discuss various issues related to nonrelativistic holography. In particular, we argue that free fermions and fermions at unitarity correspond to the same bulk theory with different choices for the nearboundary asymptotics corresponding to the source and the expectation value of one operator. We describe an extended version of nonrelativistic general coordinate invariance which is realized holographically.

DOI: 10.1103/PhysRevD.78.046003

PACS numbers: $11.25 . \mathrm{Tq}$, 03.75.Ss
\end{abstract}

\section{INTRODUCTION}

The anti-de Sitter/conformal field theory (AdS/CFT) correspondence [1-3] establishes the equivalence between a conformal field theory in flat space and a string theory in a higher-dimensional curved space. The best known example is the equivalence between $\mathcal{N}=4$ supersymmetric Yang-Mills theory and type IIB string theory in $\mathrm{AdS}_{5} \times \mathrm{S}^{5}$ space. The strong coupling limit of the field theory corresponds to the supergravity limit in which the string theory can be solved. In the recent literature, the $\mathcal{N}=4$ supersymmetric Yang-Mills theory at infinite 't Hooft coupling is frequently used as a prototype to illustrate features of strongly coupled gauge theories.

There exists, in nonrelativistic physics, another prototype of strong coupling: fermions at unitarity [4-6]. This is the system of fermions interacting through a short-ranged potential which is fine-tuned to support a zero-energy bound state. The system is scale invariant in the limit of zero-range potential. Since its experimental realizations using trapped cold atoms at the Feshbach resonance [712], this system has attracted enormous interest.

One may wonder if there exists a gravity dual of fermions at unitarity. If such a gravity dual exists, it would extend the notion of holography to nonrelativistic physics, and could potentially bring new intuition to this important strongly coupled system. Similarities between the $\mathcal{N}=4$ super-Yang-Mills theory and unitarity fermions indeed exist, the most important of which is scale invariance. The have been some speculations on the possible relevance of the universal AdS/CFT value of the viscosity/entropy density ratio [13] for unitarity fermions [14-16]. Despite these discussions, no serious attempt to construct a gravity dual of unitarity fermions has been made to date.

In this paper, we do not claim to have found the gravity dual of the unitary Fermi gas. However, we take the possible first step toward such a duality. We will construct a geometry whose symmetry coincides with the Schrödinger symmetry [17,18], which is the symmetry group of fermions at unitarity [19]. In doing so, we keep in mind that one of the main evidences for gauge/gravity duality is the coincidence between the conformal symmetry of the $\mathcal{N}=4$ field theory and the symmetry of the $\mathrm{AdS}_{5}$ space. On the basis of this geometric realization of the Schrödinger symmetry, we will be able to discuss a nonrelativistic version of the AdS/CFT dictionary-the operator-state correspondence, the relation between dimensions of operators and masses of fields, etc.

The structure of this paper is as follows. In Sec. II we give a short introduction to fermions at unitarity, emphasizing the field-theoretical aspects of the latter. We also review the Schrödinger algebra. In Sec. III we describe how Schrödinger symmetry can be embedded into a conformal symmetry in a higher dimension. We consider operator-field mapping in Sec. V. In Sec. VI we show how the conservation laws for mass, energy and momentum are realized holographically. We conclude with Sec. VII.

In this paper $d$ always refers to the number of spatial dimensions in the nonrelativistic theory, so $d=3$ corresponds to the real world.

\section{REVIEW OF FERMIONS AT UNITARITY AND SCHRÖDINGER SYMMETRY}

In this section we collect various known facts about fermions at unitarity and the Schrödinger symmetry. The goal is not to present an exhaustive treatment, but only to have a minimal amount of materials needed for later discussions. Further details can be found in [20]. We are mostly interested in vacuum correlation functions (zero temperature and zero chemical potential), but not in the thermodynamics of the system at nonzero chemical potential. The reasons are twofold: i) the chemical potential breaks the Schrödinger symmetry and ii) even at zero chemical potential there are nontrivial questions, such as the spectrum of primary operators (see below). We will comment on how chemical potential can be taken into account in Sec. VII. 
One way to arrive at the theory of unitarity fermions is to start from noninteracting fermions,

$$
\mathcal{L}=i \psi^{\dagger} \partial_{t} \psi-\frac{|\nabla \psi|^{2}}{2 m},
$$

add a source $\phi$ coupled to the "dimer" field $\psi_{\downarrow} \psi_{\uparrow}$ [21],

$$
\mathcal{L}=i \psi^{\dagger} \partial_{t} \psi-\frac{|\nabla \psi|^{2}}{2 m}+\phi^{*} \psi_{\downarrow} \psi_{\uparrow}+\phi \psi_{\uparrow}^{\dagger} \psi_{\downarrow}^{\dagger},
$$

and then promote the source $\phi$ to a dynamic field. There is no kinetic term for $\phi$ in the bare Lagrangian, but it will be generated by a fermion loop. Depending on the regularization scheme, one may need to add to (2) a counterterm $c_{0}^{-1} \phi^{*} \phi$ to cancel the UV divergence in the one-loop $\phi$ self-energy (such a term is needed in momentum cutoff regularization but not in dimensional regularization.) The theory defined by the Lagrangian (2) is UV complete in spatial dimension $2<d<4$, including the physically most relevant case of $d=3$. This system is called "fermions at unitarity," which refers to the fact that the $s$-wave scattering cross section between two fermions saturates the unitarity bound.

Another description of fermions at unitarity is in terms of the Lagrangian

$$
\mathcal{L}=i \psi^{\dagger} \partial_{t} \psi-\frac{|\nabla \psi|^{2}}{2 m}-c_{0} \psi_{\downarrow}^{\dagger} \psi_{\uparrow}^{\dagger} \psi_{\uparrow} \psi_{\downarrow},
$$

where $c_{0}$ is an interaction constant. The interaction is irrelevant in spatial dimensions $d>2$, and is marginal at $d=2$. At $d=2+\epsilon$ there is a nontrivial fixed point at a finite and negative value of $c_{0}$ of order $\epsilon$ [22]. The situation is similar to the nonlinear sigma model in $2+\epsilon$ dimensions.

In the quantum-mechanical language, unitarity fermions are defined as a system with the free Hamiltonian

$$
H=\sum_{i} \frac{\mathbf{p}_{i}^{2}}{2 m},
$$

but with a nontrivial Hilbert space, defined to contain those wave functions $\psi\left(\mathbf{x}_{1}, \mathbf{x}_{2}, \ldots ; \mathbf{y}_{1}, \mathbf{y}_{2}, \ldots\right)$ (where $\mathbf{x}_{i}$ are coordinates of spin-up particles and $\mathbf{y}_{j}$ are those of spin-down particles) which satisfy the following boundary conditions when a spin-up and a spin-down particle approach each other:

$$
\psi\left(\mathbf{x}_{1}, \mathbf{x}_{2}, \ldots ; \mathbf{y}_{1}, \mathbf{y}_{2}, \ldots\right) \rightarrow \frac{C}{\left|\mathbf{x}_{i}-\mathbf{y}_{j}\right|}+O\left(\left|\mathbf{x}_{i}-\mathbf{y}_{j}\right|\right),
$$

where $C$ depends only on coordinates other than $\mathbf{x}_{i}$ and $\mathbf{y}_{j}$. This boundary condition can be achieved by letting the fermions interact through some pairwise potential (say, a square-well potential) that has one bound state at threshold. In the limit of zero range of the potential $r_{0} \rightarrow 0$, keeping the zero-energy bound state, the two-body wave function satisfies the boundary condition (5) and the physics is universal.

Both free fermions and fermions at unitarity have the Schrödinger symmetry-the symmetry group of the Schrödinger equation in free space, which is the nonrelativistic version of conformal symmetry [19]. The generators of the Schrödinger algebra include temporal translation $H$, spatial translations $P^{i}$, rotations $M^{i j}$, Galilean boosts $K^{i}$, dilatation $D$ (where time and space dilate with different factors: $t \rightarrow e^{2 \lambda} t, \mathbf{x} \rightarrow e^{\lambda} \mathbf{x}$ ), one special conformal transformation $C$ [which takes $t \rightarrow t /(1+$ $\lambda t), \mathbf{x} \rightarrow \mathbf{x} /(1+\lambda t)]$, and the mass operator $M$. The nonzero commutators are

$$
\begin{aligned}
{\left[M^{i j}, M^{k l}\right] } & =i\left(\delta^{i k} M^{j l}+\delta^{j l} M^{i k}-\delta^{i l} M^{j k}-\delta^{j k} M^{i l}\right), \\
{\left[M^{i j}, P^{k}\right] } & =i\left(\delta^{i k} P^{j}-\delta^{j k} P^{i}\right), \\
{\left[M^{i j}, K^{k}\right] } & =i\left(\delta^{i k} K^{j}-\delta^{j k} K^{i}\right), \\
{\left[D, P^{i}\right] } & =-i P^{i}, \quad\left[D, K^{i}\right]=i K^{i}, \\
{\left[P^{i}, K^{j}\right] } & =-i \delta^{i j} M, \quad[D, H]=-2 i H, \\
{[D, C] } & =2 i C, \quad[H, C]=i D .
\end{aligned}
$$

The theory of unitarity fermions is also symmetric under an $\mathrm{SU}(2)$ group of spin rotations.

The theory of unitarity fermions is an example of nonrelativistic conformal field theories (NRCFTs). Many concepts of relativistic CFT, such as scaling dimensions and primary operators, have counterparts in nonrelativistic CFTs. A local operator $\mathcal{O}$ is said to have scaling dimension $\Delta$ if $[D, \mathcal{O}(0)]=-i \Delta \mathcal{O}(0)$. Primary operators satisfy $\left[K^{i}, \mathcal{O}(0)\right]=[C, \mathcal{O}(0)]=0$. To solve the theory of unitarity fermions at zero temperature and chemical potential is, in particular, to find the spectrum of all primary operators.

In the theory of unitarity fermions, there is a quantummechanical interpretation of the dimensions of primary operators $[20,23,24]$. A primary operator with dimension $\Delta$ and charges $N_{\uparrow}$ and $N_{\downarrow}$ with respect to the spin-up and spin-down particle numbers (the total particle numbers is $N=N_{\uparrow}+N_{\downarrow}$ ) corresponds to a solution of the zero-energy Schrödinger equation:

$$
\left(\sum_{i} \frac{\partial^{2}}{\partial x_{i}^{2}}+\sum_{j} \frac{\partial^{2}}{\partial y_{j}^{2}}\right) \psi\left(\mathbf{x}_{1}, \mathbf{x}_{2}, \ldots, \mathbf{x}_{N_{\uparrow}} ; \mathbf{y}_{1}, \mathbf{y}_{2}, \ldots, \mathbf{y}_{N_{\downarrow}}\right)=0,
$$

which satisfies the boundary condition (5) and with a scaling behavior

$$
\psi\left(\mathbf{x}_{1}, \mathbf{x}_{2}, \ldots, \mathbf{y}_{1}, \mathbf{y}_{2}, \ldots\right)=R^{\nu} \psi\left(\Omega_{k}\right),
$$

where $R$ is an overall scale of the relative distances between $\mathbf{x}_{i}, \mathbf{y}_{j}$, and $\Omega_{k}$ are dimensionless variables that are defined through the ratios of the relative distances. Equations (7) and (8) define, for given $N_{\uparrow}$ and $N_{\downarrow}$, a discrete set of possible values for $\nu$. For example, in three spatial dimensions, for $N_{\uparrow}=N_{\downarrow}=1$, there are two possible values 
for $\nu$ : 0 and -1 . For $N_{\uparrow}=2, N_{\downarrow}=1$, the lowest value for $\nu$ is $\approx-0.22728$. Each value of $\nu$ corresponds to an operator with dimension $\Delta$, which is related to $\nu$ by

$$
\Delta=\nu+\frac{d N}{2} \text {. }
$$

It has also been established that each primary operator corresponds to a eigenstate of the Hamiltonian of unitarity fermion in an isotropic harmonic potential of frequency $\omega$ $[20,23,24]$. The scaling dimension of the operator simply coincides with the energy of the state:

$$
E=\Delta \hbar \omega
$$

The first nontrivial operator is the dimer $\psi_{\downarrow} \psi_{\uparrow}$. It has dimension $\Delta=d$ in the free theory, and $\Delta=2$ in the theory of fermions at unitarity. This corresponds to the fact that the lowest energy state of two fermions with opposite spins in a harmonic potential is $d \hbar \omega$ in the case of free fermions and $2 \hbar \omega$ for unitarity fermions.

\section{EMBEDDING THE SCHRÖDINGER GROUP INTO A CONFORMAL GROUP}

To realize geometrically the Schrödinger symmetry, we first embed the Schrödinger group in $d$ spatial dimensions $\operatorname{Sch}(d)(d=3$ for the most interesting case of the unitarity Fermi gas) into the relativistic conformal algebra in $d+2$ spacetime dimensions $\mathrm{O}(d+2,2)$. The next step will be to realize the Schrödinger group as a symmetry of a $d+3$-dimensional spacetime background. That the Schrödinger algebra can be embedded into the relativistic conformal algebra can be seen from the following. Consider the massless Klein-Gordon equation in $((d+$ 1) + 1)-dimensional Minkowski spacetime,

$$
\square \phi \equiv-\partial_{t}^{2} \phi+\sum_{i=1}^{d+1} \partial_{i}^{2} \phi=0 .
$$

This equation is conformally invariant. Defining the lightcone coordinates,

$$
x^{ \pm}=\frac{x^{0} \pm x^{d+1}}{\sqrt{2}}
$$

the Klein-Gordon equation becomes

$$
\left(-2 \frac{\partial}{\partial x^{-}} \frac{\partial}{\partial x^{+}}+\sum_{i=1}^{d} \partial_{i}^{2}\right) \phi=0 .
$$

If we make an identification $\partial / \partial x^{-}=-i m$, then the equation has the form of the Schrödinger equation in free space, with the light-cone coordinate $x^{+}$playing the role of time,

$$
\left(2 i m \frac{\partial}{\partial x^{+}}+\partial_{i} \partial_{i}\right) \phi=0
$$

This equation has the Schrödinger symmetry $\operatorname{Sch}(d)$. Since the original Klein-Gordon equation has conformal symmetry, this means that $\operatorname{Sch}(d)$ is a subgroup of $\mathrm{O}(d+2,2)$.

Let us now discuss the embedding explicitly. The conformal algebra is

$$
\begin{aligned}
{\left[\tilde{M}^{\mu \nu}, \tilde{M}^{\alpha \beta}\right]=} & i\left(\eta^{\mu \alpha} \tilde{M}^{\nu \beta}+\eta^{\nu \beta} \tilde{M}^{\mu \alpha}-\eta^{\mu \beta} \tilde{M}^{\nu \alpha}\right. \\
& \left.-\eta^{\nu \alpha} \tilde{M}^{\mu \beta}\right), \\
{\left[\tilde{M}^{\mu \nu}, \tilde{P}^{\alpha}\right]=} & i\left(\eta^{\mu \alpha} \tilde{P}^{\nu}-\eta^{\nu \alpha} \tilde{P}^{\mu}\right), \\
{\left[\tilde{D}, \tilde{P}^{\mu}\right]=} & -i \tilde{P}^{\mu}, \quad\left[\tilde{D}, \tilde{K}^{\mu}\right]=i \tilde{K}^{\mu}, \\
{\left[\tilde{P}^{\mu}, \tilde{K}^{\nu}\right]=} & -2 i\left(\eta^{\mu \nu} \tilde{D}+\tilde{M}^{\mu \nu}\right),
\end{aligned}
$$

where Greek indices run $0, \ldots, d+1$, and all other commutators are equal to 0 . The tilde signs denote relativistic operators; we reserve untilded symbols for the nonrelativistic generators. We identify the light-cone momentum $\tilde{P}^{+}=\left(\tilde{P}^{0}+\tilde{P}^{d+1}\right) / \sqrt{2}$ with the mass operator $M$ in the nonrelativistic theory. We now select all operators in the conformal algebra that commute with $\tilde{P}^{+}$. Clearly these operators form a closed algebra, and it is easy to check that it is the Schrödinger algebra in $d$ spatial dimensions. The identification is as follows:

$$
\begin{gathered}
M=\tilde{P}^{+}, \quad H=\tilde{P}^{-}, \quad P^{i}=\tilde{P}^{i}, \quad M^{i j}=\tilde{M}^{i j}, \\
K^{i}=\tilde{M}^{i+}, \quad D=\tilde{D}+\tilde{M}^{+-}, \quad C=\frac{\tilde{K}^{+}}{2} .
\end{gathered}
$$

From Eqs. (15) and (16) one finds the commutators between the untilded operators to be exactly the Schrödinger algebra, Eqs. (6).

\section{GEOMETRIC REALIZATION OF THE SCHRÖDINGER SYMMETRY}

To realize the Schrödinger symmetry geometrically, we will take the AdS metric, which is invariant under the whole conformal group, and then deform it to reduce the symmetry down to the Schrödinger group. The AdS space, in Poincaré coordinates, is

$$
d s^{2}=\frac{\eta_{\mu \nu} d x^{\mu} d x^{\nu}+d z^{2}}{z^{2}} .
$$

The generators of the conformal group correspond to the following infinitesimal coordinate transformations that leave the metric unchanged:

$$
\begin{aligned}
P^{\mu}: x^{\mu} & \rightarrow x^{\mu}+a^{\mu}, \\
D: x^{\mu} & \rightarrow(1-a) x^{\mu}, \quad z \rightarrow(1-a) z, \\
K^{\mu}: x^{\mu} & \rightarrow x^{\mu}+a^{\mu}\left(z^{2}+x \cdot x\right)-2 x^{\mu}(a \cdot x)
\end{aligned}
$$

(here $x \cdot x \equiv \eta_{\mu \nu} x^{\mu} x^{\nu}$ ).

We will now deform the metric so to reduce the symmetry to the Schrödinger group. In particular, we want the metric to be invariant under $D=\tilde{D}+\tilde{M}^{+-}$, which is a linear combination of a boost along the $x^{d+1}$ direction $\tilde{M}^{+-}$and the scale transformation $\tilde{D}$, but not separately 
under $\tilde{M}^{+-}$or $\tilde{D}$. The following metric satisfies this condition:

$$
d s^{2}=-\frac{2\left(d x^{+}\right)^{2}}{z^{4}}+\frac{-2 d x^{+} d x^{-}+d x^{i} d x^{i}+d z^{2}}{z^{2}} .
$$

It is straightforward to verify that the metric (19) exhibits a full Schrödinger symmetry. From Eqs. (16) and (18) one finds that the generators of the Schrödinger algebra correspond to the following isometries of the metric:

$$
\begin{array}{ll}
P^{i}: x^{i} \rightarrow x^{i}+a^{i}, & H: x^{+} \rightarrow x^{+}+a, \quad M: x^{-} \rightarrow x^{-}+a, \\
K^{i}: x^{i} \rightarrow x^{i}-a^{i} x^{+}, & x^{-} \rightarrow x^{-}-a^{i} x^{i},
\end{array}
$$

We thus hypothesize that the gravity dual of the unitarity Fermi gas is a theory living on the background metric (19). Currently we have very little idea of what this theory is. We shall now discuss several issues related to this proposal.

(i) The mass $M$ in the Schrödinger algebra is mapped onto the light-cone momentum $P^{+} \sim \partial / \partial x^{-}$. In nonrelativistic theories the mass spectrum is normally discrete: for example, in the case of fermions at unitarity the mass of any operator is a multiple of the mass of the elementary fermion. It is possible that the light-cone coordinate $x^{-}$is compactified, which would naturally give rise to the discreteness of the mass spectrum.

(ii) In AdS/CFT correspondence the number of color $N_{c}$ of the field theory controls the magnitude of quantum effects in the string theory side: in the large $N_{c}$ limit the string theory side becomes a classical theory. The usual unitarity Fermi gas does not have this large parameter $N$; hence the dual theory probably has unsuppressed quantum effects. However, there exists an extension of the unitarity Fermi gas with $\mathrm{Sp}(2 N)$ symmetry [22,25]. The gravity dual of this theory may be a classical theory in the limit of large $N$, although with an infinite number of fields, similar to the conjectured dual of the critical $\mathrm{O}(N)$ vector model in $2+1$ dimensions [26].

(iii) We can write down a toy model in which the metric (19) is a solution to field equations. Consider the theory of gravity coupled to a massive vector field with a negative cosmological constant,

$$
\begin{aligned}
S= & \int d^{d+2} x d z \sqrt{-g}\left(R-2 \Lambda-\frac{1}{4} H_{\mu \nu} H^{\mu \nu}\right. \\
& \left.-\frac{m^{2}}{2} C_{\mu} C^{\mu}\right),
\end{aligned}
$$

where $H_{\mu \nu}=\partial_{\mu} C_{\nu}-\partial_{\nu} C_{\mu}$. One can check that Eq. (19), together with

$$
C^{-}=1 \text {, }
$$

is a solution to the coupled Einstein and Proca equations for the following choice of $\Lambda$ and $\mathrm{m}^{2}$ :

$$
\Lambda=-\frac{1}{2}(d+1)(d+2), \quad m^{2}=2(d+2) .
$$

(iv) Although the $g_{++}$metric component has $z^{-4}$ singularity at $z=0$, the metric has a plane-wave form and all scalar curvatures are finite. For example, the most singular component of the Ricci tensor, $R_{++}$, has a $z^{-4}$ singularity, as the $C_{+i+i}$ and $C_{+z+z}$ components of the Weyl tensor. However, since $g^{++}=$ 0 , any scalar constructed from the curvature tensor is regular.

(v) In terms of a dual field theory, the field $A_{\mu}$ with mass in Eq. (23) corresponds to a vector operator $O^{\mu}$ with dimension $\Delta$, which can be found from the general formula

$$
(\Delta-1)[\Delta+1+(d+2)]=2(d+2),
$$

from which $\Delta=d+3$. We thus can think about the quantum field theory as an irrelevant deformation of the original CFT, with the action

$$
S=S_{\mathrm{CFT}}+J \int d^{d+2} x O^{+} .
$$

\section{OPERATOR-FIELD CORRESPONDENCE}

Let us now discuss the relationship between the dimension of operators and masses of fields in this putative nonrelativistic AdS/CFT correspondence. Consider an operator $O$ dual to a massive scalar field $\phi$ with mass $m_{0}$. We shall assume that it couples minimally to gravity,

$$
S=-\int d^{d+3} x \sqrt{-g}\left(g^{\mu \nu} \partial_{\mu} \phi^{*} \partial_{\nu} \phi+m_{0}^{2} \phi^{*} \phi\right) .
$$

Assuming the light-cone coordinate $x^{-}$is periodic, let us concentrate only on the Kaluza-Klein mode with $P^{+}=M$. The action now becomes 


$$
\begin{aligned}
S= & \int d^{d+3} x d z \frac{1}{z^{d+3}}\left(2 i M z^{2} \phi^{*} \partial_{t} \phi-z^{2} \partial_{i} \phi^{*} \partial_{i} \phi\right. \\
& \left.-m^{2} \phi^{*} \phi\right),
\end{aligned}
$$

where the "nonrelativistic bulk mass" $m^{2}$ is related to the original mass $m_{0}^{2}$ by $m^{2}=m_{0}^{2}+2 M^{2}$. Contributions to $m^{2}$ can arise from interaction terms between $C_{\mu}$ and $\phi$, for example $\left|C^{\mu} \partial_{\mu} \phi\right|^{2},\left|C^{\mu} C^{\nu} \partial_{\mu} \partial_{\nu} \phi\right|^{2}$, etc. We therefore will treat $m^{2}$ as an independent parameter.

The field equation for $\phi$ is

$$
\partial_{z}^{2} \phi-\frac{d+1}{z} \partial_{z} \phi+\left(2 M \omega-\vec{k}^{2}-\frac{m^{2}}{z^{2}}\right) \phi=0 .
$$

The two independent solutions are

$$
\begin{gathered}
\phi_{ \pm}=z^{d / 2+1} K_{ \pm \nu}(p z), \quad p=\left(\vec{k}^{2}-2 M \omega\right)^{1 / 2}, \\
\nu=\sqrt{m^{2}+\frac{(d+2)^{2}}{4}} .
\end{gathered}
$$

As in usual AdS/CFT correspondence, one choice of $\phi_{ \pm}$ corresponds to turning a source for $O$ in the boundary theory, and another choice corresponds to a condensate of $O$. One can distinguish two cases:

(i) When $\nu \geq 1, \phi_{+}$is non-normalizable and $\phi_{-}$is renormalizable. Therefore $\phi_{+}$corresponds to the source and $\phi_{-}$to the condensate. The correlation function of $O$ is

$$
\langle O O\rangle \sim\left(\vec{k}^{2}-2 M \omega\right)^{2 \nu},
$$

which translates into the scaling dimension

$$
\Delta=\frac{d+2}{2}+\nu \text {. }
$$

(ii) When $0<\nu<1$ both asymptotics are normalizable, and there is an ambiguity in the choice of the source and condensate boundary conditions. These two choices should correspond to two different nonrelativistic CFTs. In one choice the operator $O$ has dimension $\Delta=(d+2) / 2+\nu$, and in the other choice $\Delta=(d+2) / 2-\nu$. It is similar to the situation discussed in [27].

The smallest dimension of an operator one can get is $\Delta=(d+2) / 2-\nu$ when $\nu \rightarrow 1$. Therefore, there is a lower bound on operator dimensions,

$$
\Delta>\frac{d}{2} \text {. }
$$

This bound is very natural if one remember that operator dimensions correspond to eigenvalues of the Hamiltonian in an external harmonic potential. For a system of particles in a harmonic potential, one can separate the center-ofmass motion from the relative motion. Equation (32) means that the total energy should be larger than the zero-point energy of the center-of-mass motion.
The fact that there are pairs of nonrelativistic conformal field theories with two different values of the dimensions of $O$ is a welcome feature of the construction. In fact, free fermions and fermions at unitarity can be considered as such a pair. In the theory with free fermions the operator $\psi_{\downarrow} \psi_{\uparrow}$ has dimension $d$, and for unitarity fermions, this operator has dimension 2 . The two numbers are symmetric with respect to $(d+2) / 2$ :

$$
d=\frac{d+2}{2}+\frac{d-2}{2}, \quad 2=\frac{d+2}{2}-\frac{d-2}{2} .
$$

Therefore, free fermions and fermions at unitarity should correspond to the same theory, but with different interpretations for the asymptotics of the field dual to the operator $\psi_{\downarrow} \psi_{\uparrow}$.

A similar situation exists in the case of Fermi gas at unitarity with two different masses for spin-up and spindown fermions [28]. In a certain interval of the mass ratios (between approximately 8.6 and 13.6), there exist two different scale-invariant theories which differ from each other, in our language, by the dimension of a three-body $p$-wave operator. At the upper end of the interval (mass ratio 13.6) the dimension of this operator tends to $5 / 2$ in both theories; at the lower end it has dimension $3 / 2$ in the theory with three-body resonance and $7 / 2$ in the theory without three-body resonance.

\section{TURNING ON SOURCES}

Let us now try to turn on sources coupled to conserved currents in the boundary theory. That would correspond to turning on non-normalizable modes. For the fields that enter the model action (21), the general behavior of the non-normalizable part of the metric and the field $C_{\mu}$ near $z=0$ is

$$
\begin{aligned}
d s^{2}= & -\frac{2 e^{-2 \Phi}}{z^{4}}\left(d x^{+}-B_{i} d x^{i}\right)^{2}-\frac{2 e^{-\Phi}}{z^{2}}\left(d x^{+}-B_{i} d x^{i}\right) \\
& \times\left(d x^{-}-A_{0} d x^{+}-A_{i} d x^{i}\right)+\frac{g_{i j} d x^{i} d x^{j}+d z^{2}}{z^{2}} \\
& +O\left(z^{0}\right) \\
C^{-}= & 1 .
\end{aligned}
$$

We have chosen the gauge $g_{\mu z}=0$. The non-normalizable metric fluctuations are parametrized by the functions $A_{0}$, $A_{i}, \Phi$, and $B_{i}$ of $x^{+} \equiv t$ and $x^{i}$. These functions are interpreted as background fields, on which the boundary theory exists. Following the general philosophy of AdS/ CFT correspondence, we assume that the partition function of the high-dimensional theory with the boundary condition (34) is equal to the partition function of an NRCFT in the background fields,

$$
Z=Z\left[A_{0}, A_{i}, \Phi, B_{i}, g_{i j}\right] .
$$

This partition function should be invariant with respect to a 
group of gauge transformations acting on the background fields, which we will derive.

The gauge condition $g_{\mu z}=0$ does not completely fix the metric: there is a residual gauge symmetry parametrized by arbitrary functions of $t$ and $x^{i}$ (but not of $z$ ):

$$
\begin{gathered}
t \rightarrow t^{\prime}=t+\xi^{t}(t, \mathbf{x}), \quad x^{-} \rightarrow x^{-\prime}=x^{-}+\xi^{-}(t, \mathbf{x}), \\
x^{i} \rightarrow x^{i \prime}=x^{i}+\xi^{i}(t, \mathbf{x}),
\end{gathered}
$$

and another set of infinitesimal transformations characterized by a function $\omega(t, \mathbf{x})$,

$$
z \rightarrow z^{\prime}=z-\omega(t, \mathbf{x}) z, \quad x^{\mu} \rightarrow x^{\mu \prime}=x^{\mu}+\frac{1}{2} g^{\mu \nu} \partial_{\nu} \omega .
$$

Consider first (36). Under these residual gauge transformations, the fields entering the metric (34) change in the following way:

$$
\begin{aligned}
\delta A_{0} & =\dot{\xi}^{-}-A_{0} \dot{\xi}^{t}-A_{i} \dot{\xi}^{i}-\xi^{\mu} \partial_{\mu} A_{0}, \\
\delta A_{i} & =\partial_{i} \xi^{-}-A_{0} \partial_{i} \xi^{t}-e^{\Phi} g_{i j} \dot{\xi}^{j}-\xi^{\mu} \partial_{\mu} A_{i}-A_{j} \partial_{i} \xi^{j}, \\
\delta \Phi & =\dot{\xi}^{t}-B_{i} \dot{\xi}^{i}-\xi^{\mu} \partial_{\mu} \Phi, \\
\delta B_{i} & =\partial_{i} \xi^{t}+B_{i}\left(\dot{\xi}^{t}-B_{j} \dot{\xi}^{j}\right)-\xi^{\mu} \partial_{\mu} B_{i}-B_{j} \partial_{i} \xi^{j}, \\
\delta g_{i j} & =-\left(B_{i} g_{j k}+B_{j} g_{i k}\right) \dot{\xi}^{k}-\xi^{\mu} \partial_{\mu} g_{i j}-g_{k j} \partial_{i} \xi^{k}-g_{i k} \partial_{j} \xi^{k},
\end{aligned}
$$

where $\xi^{\mu} \partial_{\mu} \equiv=\xi^{t} \partial_{t}+\xi^{i} \partial_{i}$. The residual gauge symmetry implies that the partition function of the boundary theory should be invariant under such transformations,

$$
\delta Z=0 .
$$

Can one formulate NRCFTs on background fields with this symmetry? In fact, it can be done explicitly in the theory of free nonrelativistic particles. One introduces the interaction to the background fields in the following manner:

$$
\begin{aligned}
S= & \int d t d \mathbf{x} \sqrt{g} e^{-\Phi}\left[\frac{i}{2} e^{\Phi}\left(\psi^{\dagger} D_{t} \psi-D_{t} \psi^{\dagger} \psi\right)\right. \\
& -\frac{g^{i j}}{2 m} D_{i} \psi^{\dagger} D_{j} \psi-\frac{B^{i}}{2 m}\left(D_{t} \psi^{\dagger} D_{i} \psi^{\dagger}+D_{i} \psi^{\dagger} D_{t} \psi\right) \\
& \left.-\frac{B^{2}}{2 m} D_{t} \psi^{\dagger} D_{t} \psi\right],
\end{aligned}
$$

where $g^{i j}$ is the inverse matrix of $g_{i j}, g \equiv \operatorname{det}\left|g_{i j}\right|, B^{i} \equiv$ $g^{i j} B_{i}, B^{2} \equiv B^{i} B_{i}$, and $D_{\mu} \psi \equiv \partial_{\mu} \psi-i m A_{\mu} \psi$. One can verify directly that the action (40) is invariant under the transformations (38), if $\psi$ transforms as

$$
\delta \psi=i m \xi^{-} \psi-\xi^{\mu} \partial_{\mu} \psi
$$

In fact, this invariance is an extension of the general coordinate invariance previously discussed in [29]. The invariance found in [29] corresponds to restricting $\Phi=$ $B_{i}=\xi^{t}=0$ in all formulas.
To linear order in external field, the action is

$$
\begin{aligned}
S= & S[0]+\int d t d \mathbf{x}\left(A_{0} \rho+A_{i} j^{i}+\Phi \epsilon+B_{i} j_{\epsilon}^{i}\right. \\
& \left.+\frac{1}{2} h_{i j} \Pi^{i j}\right),
\end{aligned}
$$

and from Eq. (40) one reads out the physical meaning of the operators coupled to the external sources:

(i) $h_{i j}$ is coupled to the stress tensor $\Pi^{i j}$,

(ii) $A_{\mu}$ is coupled to the mass current $(\rho, \mathbf{j})$,

(iii) $\left(\Phi, B_{i}\right)$ are coupled to the energy current $\left(\epsilon, \mathbf{j}_{\epsilon}\right)$.

The invariance of the partition function with respect to the gauge transformations (38) leads to an infinite set of Takahashi-Ward identities for the correlation functions. The simplest ones are for the one-point functions. The fact that the group of invariance includes gauge transformation of $A_{\mu}: \delta A_{\mu}=\partial_{\mu} \xi^{-}$guarantees the conservation of mass. The fact that the linear parts in the transformation laws for $\Phi$ and $B_{i}$ look like a gauge transformation, $\delta \Phi=$ $\dot{\xi}^{t}+\cdots$ and $\delta B_{i}=\partial_{i} \xi^{t}+\cdots$ leads to energy conservation in the absence of external fields:

$$
\partial_{t}\left\langle\frac{\partial \ln Z}{\partial \Phi}\right\rangle+\left.\partial_{i}\left\langle\frac{\partial \ln Z}{\partial B_{i}}\right\rangle\right|_{A_{\mu}=\Phi=B_{i}=h_{i j}=0}=0
$$

Energy is not conserved in a general background (which is natural, since the background fields exert external forces on the system). Similarly, momentum conservation $\partial_{t} j^{i}+$ $\partial_{j} \Pi^{i j}=0$ (and the fact that momentum density coincides with mass current) is related to terms linear in $\xi^{i}$ in $\delta A_{i}$ and $\delta g_{i j}: \delta A_{i}=-\dot{\xi}^{i}+\cdots, \delta g_{i j}=-\partial_{i} \xi^{j}-\partial_{j} \xi^{i}+\cdots$.

Let us now turn to the transformations (37), under which

$$
\delta \Phi=2 \omega, \quad \delta g_{i j}=-2 \omega g_{i j} .
$$

The invariance of the partition function with respect to this transformation implies

$$
2 \epsilon=\Pi_{i}^{i},
$$

which is the familiar relationship between energy and pressure,

$$
E=\frac{d}{2} P V,
$$

valid for free gas as well as for Fermi gas at unitarity. The action (40) is not invariant under (44), but it can be made so by replacing the "minimal coupling" by a "conformal coupling" to external fields. Therefore, the proposed holography is consistent with conservation laws and the universal thermodynamic relation between energy and pressure. 


\section{CONCLUSION}

The main goal of the paper is to construct a geometry with the symmetry of the Schrödinger group. The existence of such a geometrical realization makes it possible to discuss the possibility of a dual description of Fermi gas at unitarity at a concrete level. It remains to be seen if holography is a notion as useful in nonrelativistic physics as it is for relativistic quantum field theories. At the very least, one should expect holography to provide toy models with Schrödinger symmetry.

In this paper we have considered only the properties of the vacuum correlation functions. In order to construct the gravity dual of the finite-density ground state, about which a lot is known both experimentally and theoretically, one should turn on a background $A_{0}$ in the metric (34). Superfluidity of the system should be encoded in the condensation of the scalar field $\psi_{\uparrow} \psi_{\downarrow}$ (whose dimension is 2 in the case of unitarity fermions, cf. [30,31]). It would be interesting to find black-hole metrics which realize nonrelativistic hydrodynamics and superfluid hydrodynamics. We defer this problem to future work.

\section{ACKNOWLEDGMENTS}

The author thanks A. Karch and Y. Nishida for discussions leading to this work, and S. Hartnoll, V. Hubeny, D. Mateos, H. Liu, K. Rajagopal, M. Rangamani, S. Shenker, and M. Stephanov for valuable comments. This work is supported, in part, by DOE Grant No. DEFG02-00ER41132.

Note added-After this work was completed, J. McGreevy informed the author that he and K. Balasubramanian have also obtained the metric (19) and determined that it has nonrelativistic conformal symmetry [32].
[1] J. Maldacena, Adv. Theor. Math. Phys. 2, 231 (1998); Int. J. Theor. Phys. 38, 1113 (1999).

[2] S. S. Gubser, I. R. Klebanov, and A. M. Polyakov, Phys. Lett. B 428, 105 (1998).

[3] E. Witten, Adv. Theor. Math. Phys. 2, 253 (1998).

[4] D. M. Eagles, Phys. Rev. 186, 456 (1969).

[5] A. J. Leggett, Modern Trends in the Theory of Condensed Matter (Springer, Berlin, 1980).

[6] P. Nozières and S. Schmitt-Rink, J. Low Temp. Phys. 59, 195 (1985)

[7] K. M. O'Hara et al., Science 298, 2179 (2002).

[8] C. A. Regal, M. Greiner, and D. S. Jin, Phys. Rev. Lett. 92, 040403 (2004).

[9] M. Bartenstein et al., Phys. Rev. Lett. 92, 120401 (2004).

[10] M. W. Zwierlein et al., Phys. Rev. Lett. 92, 120403 (2004).

[11] J. Kinast et al., Phys. Rev. Lett. 92, 150402 (2004).

[12] T. Bourdel et al., Phys. Rev. Lett. 93, 050401 (2004).

[13] P. Kovtun, D. T. Son, and A. O. Starinets, Phys. Rev. Lett. 94, 111601 (2005); A. Buchel and J. T. Liu, Phys. Rev. Lett. 93, 090602 (2004); G. Policastro, D. T. Son, and A. O. Starinets, Phys. Rev. Lett. 87, 081601 (2001).

[14] B. A. Gelman, E. V. Shuryak, and I. Zahed, Phys. Rev. A 72, 043601 (2005).

[15] T. Schäfer, Phys. Rev. A 76, 063618 (2007).

[16] A. Turlapov et al., J. Low Temp. Phys. 150, 567 (2008).
[17] C. R. Hagen, Phys. Rev. D 5, 377 (1972).

[18] U. Niederer, Helv. Phys. Acta 45, 802 (1972).

[19] T. Mehen, I. W. Stewart, and M. B. Wise, Phys. Lett. B 474, 145 (2000).

[20] Y. Nishida and D. T. Son, Phys. Rev. D 76, 086004 (2007).

[21] Y. Nishida and D. T. Son, Phys. Rev. Lett. 97, 050403 (2006).

[22] P. Nikolić and S. Sachdev, Phys. Rev. A 75, 033608 (2007).

[23] F. Werner and Y. Castin, Phys. Rev. A 74, 053604 (2006).

[24] S. Tan (unpublished).

[25] M. Y. Veillette, D. E. Sheehy, and L. Radzihovsky, Phys. Rev. A 75, 043614 (2007).

[26] I. R. Klebanov and A. M. Polyakov, Phys. Lett. B 550, 213 (2002).

[27] I. R. Klebanov and E. Witten, Nucl. Phys. B556, 89 (1999).

[28] Y. Nishida, D. T. Son, and S. Tan, Phys. Rev. Lett. 100, 090405 (2008).

[29] D. T. Son and M. Wingate, Ann. Phys. (N.Y.) 321, 197 (2006).

[30] S. A. Hartnoll, C.P. Herzog, and G. T. Horowitz, arXiv:0803.3295.

[31] S.S. Gubser, arXiv:0803.3483.

[32] K. Balasubramanian and J. McGreevy, arXiv:0804.4053. 